\title{
Expression of Yes-associated Protein in Oral Squamous Cell Carcinoma
}

\author{
Pankaj Shelke ${ }^{1}$, Gargi S Sarode ${ }^{2}$, Sachin C Sarode ${ }^{3}$, Nikunj Maniyar ${ }^{4}$, Ghevaram Prajapati ${ }^{5}$, Shankargouda Patil ${ }^{6}$
}

\begin{abstract}
Aim:Though the exact mechanism of yes-associated protein (YAP) in tumorigenesis is not well understood, studies have shown that YAP plays an imperative role in cancer advancement via the Hippo signaling pathway. The purpose of the present study was to appraise the clinicopathological correlation of YAP expression in various grades and stages along with different parameters like tumor size and nodal metastasis in oral squamous cell carcinoma (OSCC).

Materials and methods: Twenty-seven surgically excised specimens from patients with OSCC were selected for the study. Grading of the OSCC specimens was done according to Broder's grading system and staging had been done using tumor-node-metastasis (TNM) system by American Joint Committee on Cancer (AJCC). Comparative analysis of YAP expression and various aforementioned parameters of OSCC was performed and statistically analyzed.

Results: Differences in the expression of YAP was observed among well differentiated and moderately differentiated OSCC, with increased YAP expression with successive grades but was statistically insignificant. On evaluating YAP expression among various stages of OSCC, we observed that stages I, II, and IV demonstrated a weak expression of YAP, while stage III showed a strong expression but the differences were insignificant. Insignificant differences were also noted in YAP expression between different tumor sizes, while significant differences were observed between different nodal statuses.

Conclusion: Our results suggest that YAP could be responsible for extensive proliferation and invasiveness of OSCC.

Keywords: Oral cancer, Oral squamous cell carcinoma, Prognostic marker, Yes-associated protein.

The Journal of Contemporary Dental Practice (2019): 10.5005/jp-journals-10024-2633
\end{abstract}

\section{INTRODUCTION}

Oral cancer, being a serious issue worldwide, has been positioned among the top three types of cancer in the country. ${ }^{1}$ Because of social, cultural, topographical aspects, and existence of different addictive practices, the occurrence of oral cancer is very common. ${ }^{2}$ Irrespective of disposal of the latest diagnostic and therapeutic approaches regarding diagnosis of squamous cell carcinoma of oral cavity (OSCC) and its treatment, the survival of these OSCC patients has not revealed much development. ${ }^{3}$ Millions of people capitulate to OSCC annually. ${ }^{4}$ Being a multifactorial disorder, numerous pathogeneses have been proposed till date explaining the development of OSCC. Understanding the molecular mechanisms of carcinogenesis will offer precious knowledge on diagnosis and prognosis, as well as help in developing a novel therapy. Understanding the biological behavior of cancer is of utmost value to settle on the most apposite therapeutic strategy and ascertain the prognosis of the lesion, which can be facilitated by identification of prognostic markers. Innovative diagnostic techniques like polymerization chain reaction (PCR), enzyme-linked immunosorbent assay (ELISA), cell culture, and immunohistochemistry have been recommended to determine prognostic markers in OSCC. YAP is one such prognostic marker that is involved in carcinogenesis of various malignancies, including OSCC. ${ }^{5}$

YAP, a strategic controller of size of the organs, is involved in the Hippo pathway as a nuclear effecter. The Hippo signaling pathway panels the poise among cellular proliferation and apoptosis. ${ }^{6}$ It consists of a regulatory serine-threonine kinase module and a transcriptional module, the kinase module including mammalian STE20-like protein kinase 1 (MST1) and MST2, large tumor suppressor 1 (LATS1) and LATS2, together with the adaptor proteins salvador homologue 1 (SAV1), MOB kinase activator 1A (MOB1A), \begin{tabular}{l}
\hline${ }^{1-5}$ Department of Oral Pathology and Microbiology, Dr D. Y. Patil Dental \\
College and Hospital, Dr D.Y. Patil Vidyapeeth, Pune, Maharashtra, India \\
${ }^{6}$ Department of Maxillofacial Surgery and Diagnostic Sciences, Division \\
of Oral Pathology, College of Dentistry, Jazan University, Kingdom of \\
Saudi Arabia \\
Corresponding Author: Gargi S Sarode, Department of Oral Pathology \\
and Microbiology, Dr D. Y. Patil Dental College and Hospital, Dr D.Y. \\
Patil Vidyapeeth, Pune, Maharashtra, India, Phone: +91 9823871462, \\
e-mail: gargi14@gmail.com \\
How to cite this article: Shelke P, Sarode GS, et al. Expression of Yes- \\
associated Protein in Oral Squamous Cell Carcinoma. J Contemp Dent \\
Pract 2019;20(8):887-892. \\
Source of support: Nil \\
Conflict of interest: None \\
\hline \hline
\end{tabular}

and $\mathrm{MOB1B}{ }^{7}$ These inhibitory kinase modules regulate tissue growth by suppressing the transcription module such as the YAP-1 (YAP1) and transcriptional coactivator with PDZ-binding motif Tafazzin (TAZ). ${ }^{8}$ YAP is often considered as a mammalian ortholog of Drosophila Yorkie (Yki) that endows with a negative feedback to direct the Hippo pathway. It plays a role as transcriptional coactivator/corepressor in controlling cellular growth, proliferation, and apoptosis. Tumorigenesis and distant metastasis-related activities are augmented via transcriptional enhanced associate domain (TEAD) interaction that is domain by YAP. ${ }^{9}$ YAP1 is shown to regulate biological events like epithelial-mesenchymal transition (EMT), cancer cell migration, and cancer stem cell properties. YAP has also proved to play a major role in cancer cell proliferation and alteration, their migration, and invasion. ${ }^{10}$ YAP has been archived as a bonafide oncogene in the diagnosis of cancer with its intensification 
and overexpression been seen in several human tumors and mouse models of cancer. Minor complexes have been recognized that interrupt the YAP1-TEAD compound or block the binding purpose of WW domains. ${ }^{11}$ These minor particles characterize principal complexes for the improvement of treatments for cancer patients, who port augmented or overexpressed YAP oncogene. ${ }^{5}$

Clinging to the aforementioned aspects of YAP, we hypothesized that OSCC may show an increased expression of YAP in the tumor cells and the expression may increase with the grade and stage of the cancer. The present article is an attempt to evaluate the expression of YAP in OSCC cells with clinicopathological parameters.

\section{Materials and Methods}

\section{Human Tissue Samples}

Study samples were retrieved from histopathologically diagnosed archival formalin-fixed and paraffin-embedded excisional OSCC tissue samples (2013-2018) at the Department of Oral Pathology and Microbiology, Dr D. Y. Patil Dental College and Hospital, Pune. A total of 28 biopsy specimens (2 slides each) of OSCC were included in the study.

\section{Antibodies and Immunohistochemistry}

The tissue specimens were subject to immunohistochemical analysis. Five $\mu \mathrm{m}$ thick paraffin sections were taken on glass slides with poly-l-lysine coating and stained immunohistochemically using diluted YAP antibody (1:200 in antibody diluents). The tissue sections were treated in protease XXV at $1 \mathrm{mg} / \mathrm{mL}$ PBS (Lab Vision Catalog \# AP-9004) for 5 minutes at $37^{\circ} \mathrm{C}$. The primary antibody incubation time was 30 minutes at room temperature. The heatinduced epitope retrieval (HIER) method was used to retrieve the antigen. Then immunohistochemical staining of the section by the primary antibody YAP (C-20): sc558 was carried out. Counter staining was done with Mayer's hematoxylin. The slides were then observed under a light microscope for immunohistochemical grading using thyroid carcinoma as a control (Fig. 1).

\section{Method of Data Analysis}

The expression of YAP cancerous tissue was scored by a semiquantitative method. Scoring was based on (a) the intensity of the immunostaining in the malignant cells $(0=$ absent,

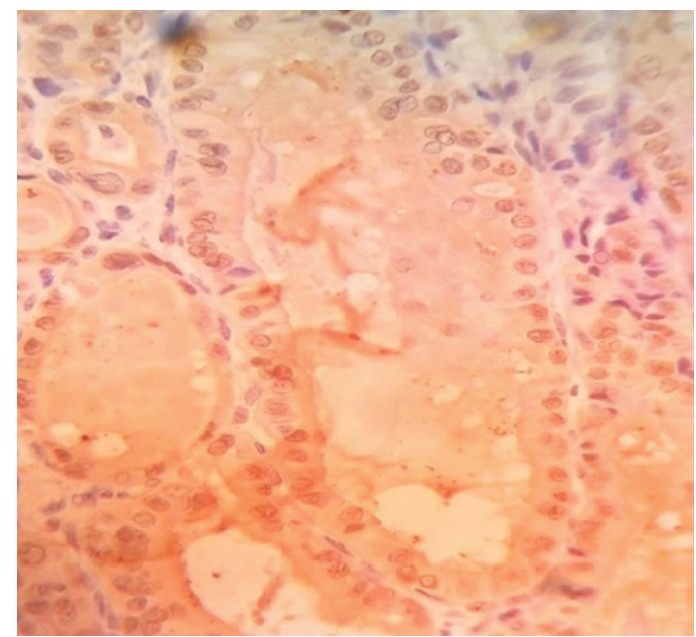

Fig. 1: Photomicrograph showing strong expression of YAP in thyroid carcinoma (control) (total magnification $\times 400$; IHC staining)
$1=$ weak, $2=$ moderate, $3=$ strong, and $4=$ very strong) and (b) the percentage of positive malignant cells $(0=0 \%$ positive cells, $1=<25 \%$ positive cells, $2=25-50 \%$ positive cells, $3=50-75 \%$ positive cells, and $4=>75 \%$ positive cells). The final immunostaining score was determined by the sum of $(a)+(b)$. Final scores ranged from $0-8(0=$ absent, $1-4=$ weak, and $5-8=$ strong $)$. The score of YAP expression in OSCC was assessed and categorized into three groups: (-) negative, $(+)$ weakly to moderately positive, and $(++)$ strongly positive. The evaluation of IHC slides was done by two competent investigators and kappa analysis was applied to analyze the degree of agreement.

\section{Results}

\section{Demographic Findings}

A total of 28 OSCC cases were included in the study. The age of patients with OSCC ranged from $46-80$ years (mean of $60.92 \pm 8.17$ years) with a male predilection $[19 / 28(67.86 \%)]$ observed in relation to females [09/28 (32.14\%)]. The mandible [25/28 (89.28\%)] was more commonly affected than the maxilla [2/28 (7.14\%)] followed by the tongue $[1 / 28(3.57 \%)]$. Out of the 25 mandibular cases, $2(8 \%)$ involved the gingiva, $3(12 \%)$ involved the buccal vestibule, $4(16 \%)$ involved the gingivobuccal complex, 12 (48\%) involved the buccal mucosa, 1 (4\%) involved the buccal and labial vestibule and alveolar region, 1 (4\%) involved the gingivobuccal complex, alveolus, and floor of the mouth, $1(4 \%)$ involved the alveolar region, and $1(4 \%)$ involved the alveolus and floor of the mouth. From maxillary cases, 1 (50\%) was associated with maxillary tuberosity and the other one involved gingivobuccal complex [01/02 (50\%)]. Only single case was present on the right part of the tongue (3.57\%). Most common clinical appearance of the lesion was ulcero-proliferative growth [17/28 (60.71\%)] followed by exophytic growth in $2(7.14 \%)$ cases, endophytic in $2(7.14 \%)$ cases, and draining sinus in 2 (7.14\%) cases. One case $(3.57 \%)$ each of erythroplakic lesion, ulcerative lesion, and trismus with cauliflower like growth was found and, in 2 cases, the feature was not mentioned. On histopathologic reexamination, 15 (53.57\%) cases were found to be well differentiated while $13(46.42 \%)$ cases were moderately differentiated OSCC using Broder's grading system and TNM (AJCCS) had been used for staging the cases (Tables 1 and 2).

\section{Comparison of YAP Expression in Different Grades of OSCC}

In the current study, YAP expression in OSCC was assessed in all the grades and stages of OSCC (Fig. 2). On the one hand, in OSCC, welldifferentiated OSCC showed a weak expression in $11 / 15$ cases (73.33\%), while a strong expression was observed in 4/15 (26.66\%) cases for YAP (Fig. 2A). On the other hand, moderately differentiated OSCC showed a strong expression in $7 / 13$ (53.84\%) cases (Fig. 2B) and a weak expression in $6 / 13(46.15 \%)$ cases (Table 3 and Fig. 3). Thus, YAP expression significantly increased with successive histologic grades of OSCC.

\section{Comparison of Expression of YAP in Different Stages of OSCC}

The expression of YAP in different clinical stages of OSCC retrieved from the data was compared. A total of 28 cases were included in

Table 1: Distribution of OSCC cases according to histopathological types

\begin{tabular}{lcc}
\hline Type & $N$ & $\%$ \\
\hline Total (28) & & \\
Well differentiated & 15 & 53.57 \\
Moderately differentiated & 13 & 46.42 \\
\hline
\end{tabular}


Table 2: Demographic data and patients characteristics of OSCC

\begin{tabular}{|c|c|c|}
\hline Sno & Patients characteristics & Number \\
\hline 1 & Total (28) & \\
\hline \multirow[t]{3}{*}{2} & Age (years) & \\
\hline & Mean SD & $60.92 \pm 8.17$ years \\
\hline & Range & $46-80$ years \\
\hline \multirow[t]{3}{*}{3} & Gender & \\
\hline & Male & $19(67.86 \%)$ \\
\hline & Female & $09(32.14 \%)$ \\
\hline \multirow[t]{14}{*}{4} & Site & \\
\hline & a Maxilla [02 (7.14\%)] & \\
\hline & Maxillary tuberosity region & $01(50 \%)$ \\
\hline & Gingivobuccal complex & $01(50 \%)$ \\
\hline & b Mandibular region [25 (89.28\%)] & \\
\hline & Gingiva & $02(8 \%)$ \\
\hline & Buccal vestibule & $03(12 \%)$ \\
\hline & Gingivobuccal complex & $04(16 \%)$ \\
\hline & Buccal mucosa & $12(48 \%)$ \\
\hline & $\begin{array}{l}\text { Buccal and labial vestibule and } \\
\text { alveolusalveolar region }\end{array}$ & $01(4 \%)$ \\
\hline & $\begin{array}{l}\text { Gingivobuccal complex, } \\
\text { alveolus, floor }\end{array}$ & $01(4 \%)$ \\
\hline & Alveolar region & $01(4 \%)$ \\
\hline & Alveolus and floor & $01(4 \%)$ \\
\hline & c Tongue $[01(3.57 \%)]$ & \\
\hline \multirow[t]{9}{*}{5} & Clinical appearance & \\
\hline & Erythroplakia & $01(3.57 \%)$ \\
\hline & Endophytic growth & $02(7.14 \%)$ \\
\hline & Ulcerative & $01(3.57 \%)$ \\
\hline & Exophytic & $02(7.14 \%)$ \\
\hline & Ulceroproliferative & $17(60.71 \%)$ \\
\hline & Trismus, cauliflower like growth & $01(3.57 \%)$ \\
\hline & Draining sinus & $02(7.14 \%)$ \\
\hline & Not mentioned & $02(7.14 \%)$ \\
\hline \multirow[t]{9}{*}{6} & Radiological features & \\
\hline & Bone loss & $03(10.71 \%)$ \\
\hline & Bone erosion & $02(7.14 \%)$ \\
\hline & Pathological fracture & $02(7.14 \%)$ \\
\hline & Extension of the lesion till RMT (CT) & $02(7.14 \%)$ \\
\hline & $\begin{array}{l}\text { Involvement of floor of the mouth } \\
\text { and alveolar region (CT) }\end{array}$ & $02(7.14 \%)$ \\
\hline & Enlarged lymph nodes (CT) & $01(3.57 \%)$ \\
\hline & $\begin{array}{l}\text { Radiolucency with maxillary sinus } \\
\text { region invading the orbital floor }\end{array}$ & $01(3.57 \%)$ \\
\hline & Not mentioned & $15(53.57 \%)$ \\
\hline
\end{tabular}

the present study. Three (10.71\%) cases were presented as stage I, 10 (35.71\%) cases showed stage II, and $13(48.42 \%)$ cases revealed stage III involvement, while one case (3.75\%) each belonged to stages IVa and IVb. The staging status was retrieved from the patients' data from the Department of Oral Surgery. Two (66.66\%) cases of stage I showed a weak expression, while one (33.33\%) case displayed a strong expression. Out of 10 stage II cases, 8 (80\%) showed a weak expression, while $2(20 \%)$ revealed a strong expression. Out of 13 $(48.42 \%)$ stage III cases, 5 (38.46\%) cases had a weak expression, while $8(61.53 \%)$ cases had a strong expression. The single case each $(3.75 \%)$ of stages IVa and b had a weak expression (Table 4 and Fig. 4). The maximum number of stage III cases showed strong YAP expression but there was no statistically significant difference between YAP expression in different stages.

\section{Comparison of Expression of YAP in Various Tumor Sizes of OSCC Cases}

The expression of YAP in various tumor sizes was compared in OSCC. The clinical staging from the existing data was used for comparison. There were six cases with T1, one with a strong expression, while five with a weak expression for YAP. Cases with T2 were 18 (\%), of which 9 (50\%) showed weak while remaining showed strong YAP expression (50\%). Two cases (\%) with T3 showed a strong expression. There were only two cases (3.57\%) with T4a, which showed a weak expression for YAP (Table 5). The maximum number of cases with T2 showed strong YAP expression but there is no statistically significant difference between YAP expression and different tumor sizes.

\section{Comparison of Expression of YAP and Nodal Metastasis in OSCC}

In the present study, YAP expression in various nodal statuses has been studied. There were a total of 13 OSCC cases showing nodal metastasis [N1: 12 (42.85\%); N3: 1 (3.57\%)]. Out of them, 12 cases belonged to stage III, while the single case belonged to stage IVb. It has been found that out of 15 (53.57\%) N0 cases, 12 (80\%) cases showed a weak expression, while remaining three $(20 \%)$ expressed strong staining for YAP. Out of $12(42.85 \%)$ with N1 nodal status, 4 (33.33\%) expressed YAP weakly, while $8(66.66 \%)$ expressed it strongly. The single case (3.57\%) with N3 nodal status showed a weak expression for YAP (Table 6 and Fig. 5). There is a statistically significant difference between YAP expression and nodal metastasis. YAP expression increases with higher nodal status.

\section{Discussion}

The Hippo signaling pathway that is significantly important in controlling organ growth and development, stem cell function, tumor suppression, and regeneration was first discovered in the 1990s on Drosophila for mutants exhibiting tissue overgrowth. ${ }^{12-14}$ The Hippo flagging pathway has an expansive number of mammalian homologues, for example, Lats1/2, Mob1, Mst1/2, and Sav1, in mammalian are homologues of Hpo, Sav, Wts, and Mats, individually; both YAP and TAZ are homologues of Yki. ${ }^{14}$ The present investigation was focused on the expression of YAP in OSCC and was aimed to uncover that YAP assumes a part in tumorigenesis and might be helpful as a free prognostic marker in OSCC. In spite of the fact that it stays questionable whether this conduct is because of a tissue-particular capacities of the Hippo pathway or just because of rare investigations of this pathway in different tissues. Significant numbers of studies have shown that the Hippo pathway performs a crucial role in tumor progression. In addition to enhancement of disease cells' development, down-regulation of the Hippo pathway makes growth cells impervious to some chemotherapeautic drugs. ${ }^{15}$ These discoveries raise the likelihood that remedial intercession of the Hippo flagging pathway may enhance momentum treatment methodologies and models and end up being recipient. The significant focuses of the YAP and TAZ are the homologous individual from the TEAD protein family (TEAD1-4) that control cell contact hindrance, epithelial-mesenchymal transformation (EMT), oncogenic alteration, and apoptotic temperance. The association 

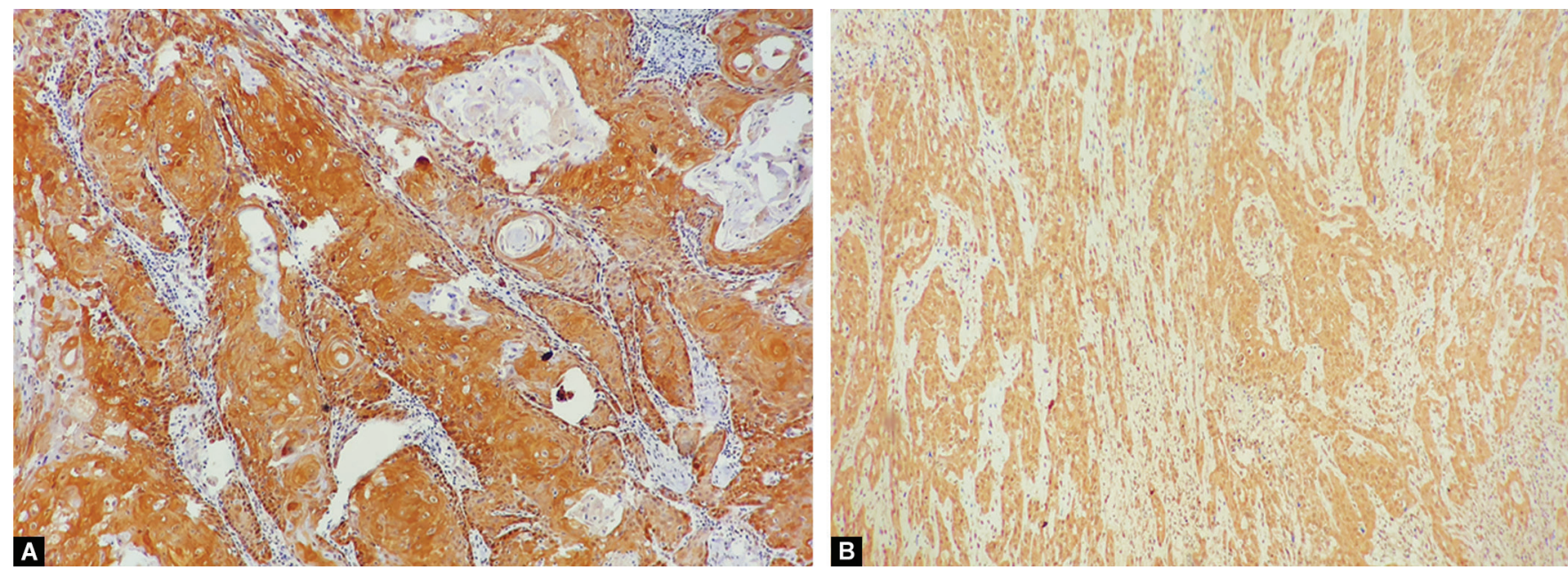

Figs 2A and B: Photomicrograph showing strong expression of YAP in different grades of OSCC; (A) Well-differentiated OSCC; (b) Moderately differentiated OSCC (total magnification $\times 400$; IHC staining)

Table 3: YAP expression in different grades of OSCC

\begin{tabular}{|c|c|c|c|c|c|c|c|}
\hline \multirow[b]{2}{*}{ OSCC grade } & \multirow[b]{2}{*}{$N(\%)$} & \multicolumn{3}{|c|}{ YAP expression } & \multirow[b]{2}{*}{ Chi-square value } & \multirow[b]{2}{*}{$p$ value } & \multirow[b]{2}{*}{ Significance } \\
\hline & & Negative & Weak & Strong & & & \\
\hline Well differentiated & $15(53.57)$ & 0 & $11(73.33)$ & $04(26.66)$ & 2.1569 & 0.1419 & Insignificant \\
\hline Moderately differentiated & $13(46.43)$ & 0 & $06(7.69)$ & $07(92.3)$ & & & \\
\hline
\end{tabular}

of the TEAD with the TB spaces prompts enactment of downstream quality transcripts, for example, CTGF and Cyr61. To demolish their capacity to advance cell expansion and tumorigenesis, upsetting the official of YAP or TAZ to TEAD by hereditary tack can be utilized as a remedial regime. ${ }^{16}$

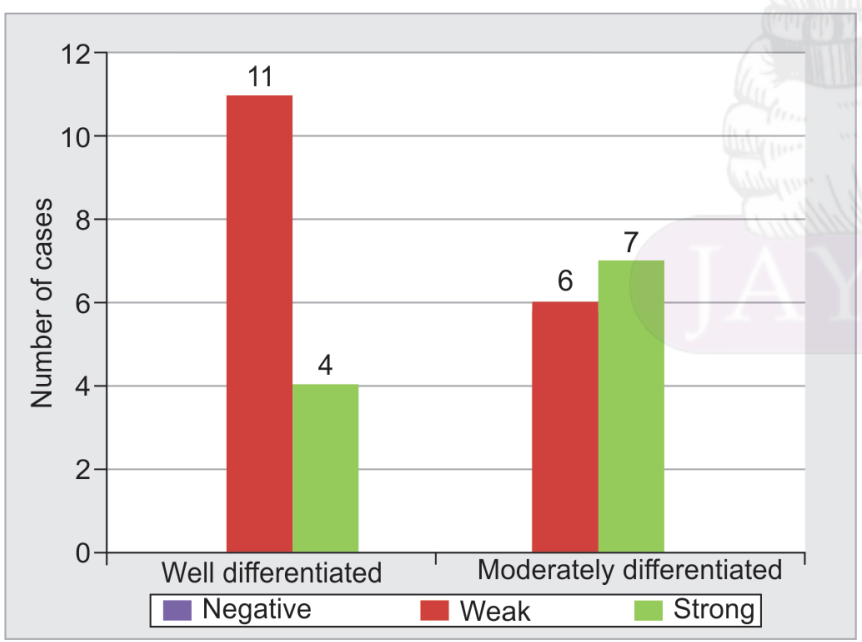

Fig. 3: YAP expression in different grades of OSCC
The present study used immunohistochemistry to compute and evaluate expression criterions of YAP in OSCC, between its different grades and stages, to scrutinize the mantle of this molecule in oral cancer development and progression. In this study, 28 OSCC cases were included with patient's age ranging from $46-80$ years (mean of $60.92 \pm 8.17$ years). Thyroid carcinoma that shows a strong expression of YAP was used as a positive control. On the one hand, in the present study, YAP expression was assessed in well-differentiated and moderately differentiated grades and all the stages of OSCC. We observed a weak expression of YAP in $73.33 \%$ of well-differentiated OSCC, while a strong expression was observed in $26.66 \%$ cases for YAP. On the other hand, moderately differentiated OSCC showed a strong expression in $53.84 \%$ cases, while a weak expression was seen in $46.15 \%$ cases. In a study piloted by Zhang et al., ${ }^{17}$ they perceived increased YAP expression in OSCC samples and deduced that YAP was more recurrently expressed in the invasive margin of the tumor than to its interior. In contrast to our results, Yuan and Tomlinson ${ }^{18}$ analyzed the expression of YAP in relation to traditional prophetic indices of breast carcinoma to show no noteworthy correspondence with tumor grade; however, there was a propensity toward superior incidence of loss in the higher grade tumors, with $55.6 \%$ (5 of 9) grade I, 30.8\% (8 of 26) grade II, and only $36.4 \%$ (24 of 66 ) grade III retaining YAP expression

Table 4: Comparison of expression of YAP in different stages of OSCC

\begin{tabular}{|c|c|c|c|c|c|c|c|}
\hline \multirow[b]{2}{*}{ OSCC stages } & \multirow[b]{2}{*}{$N(\%)$} & \multicolumn{3}{|c|}{ YAP expression } & \multirow[b]{2}{*}{ Chi-square value } & \multirow[b]{2}{*}{$p$ value } & \multirow[b]{2}{*}{ Significance } \\
\hline & & Negative & Weak (\%) & Strong (\%) & & & \\
\hline I & $03(10.71)$ & 0 & 2 (66.66) & $1(33.33)$ & 2.8449 & 0.24112 & Insignificant \\
\hline II & $10(35.71)$ & 0 & $8(80)$ & $2(20)$ & & & \\
\hline III & $13(48.42)$ & 0 & $5(38.46)$ & $8(61.53)$ & & & \\
\hline IVa & $01(3.75)$ & 0 & $1(100)$ & $0(0)$ & & & \\
\hline $\mathrm{IVb}$ & 01 (3.75) & 0 & $1(100)$ & $0(0)$ & & & \\
\hline
\end{tabular}




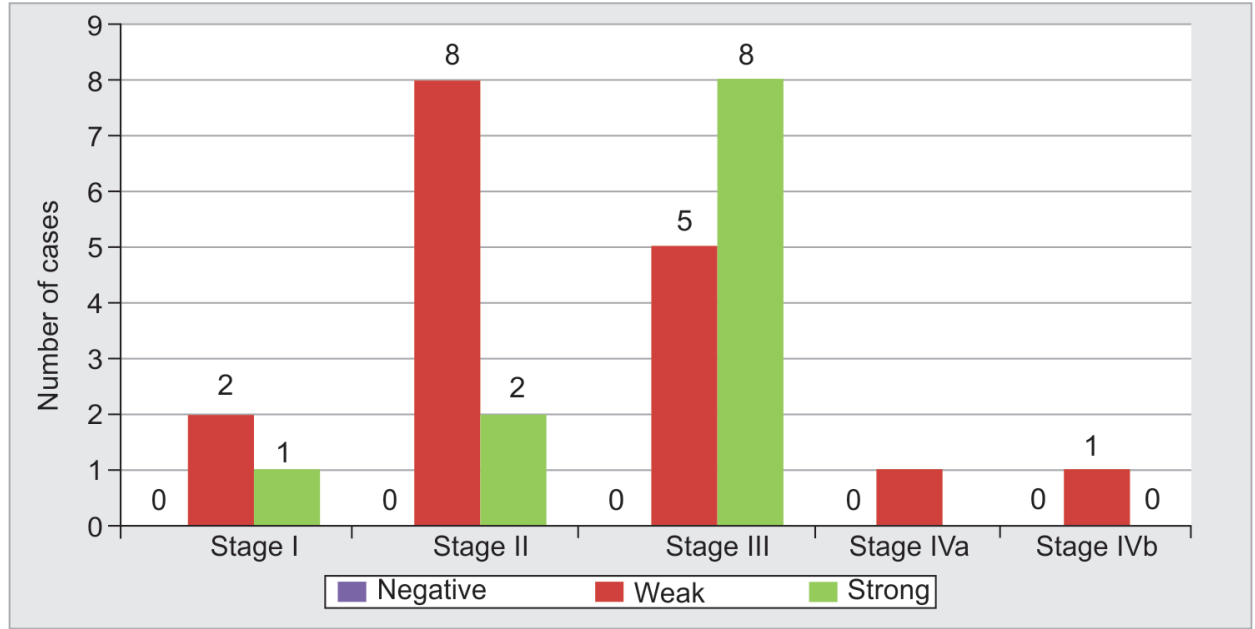

Fig. 4: Comparison of expression of YAP in different stages of OSCC

Table 5: Comparison of expression of YAP in various tumor sizes of OSCC cases

\begin{tabular}{|c|c|c|c|c|c|c|c|}
\hline \multirow[b]{2}{*}{ Tumor size } & \multirow[b]{2}{*}{$N(\%)$} & \multicolumn{3}{|c|}{ YAP expression } & \multirow[b]{2}{*}{ Chi-square value } & \multirow[b]{2}{*}{$p$ value } & \multirow[b]{2}{*}{ Significance } \\
\hline & & Negative & Weak (\%) & Strong (\%) & & & \\
\hline T1 & $06(21.42)$ & 0 & $5(83.33)$ & $1(16.66)$ & 0.6728 & 0.412086 & Insignificant \\
\hline $\mathrm{T} 2$ & $18(64.28)$ & 0 & $9(50)$ & $9(50)$ & & & \\
\hline T3 & $02(7.14)$ & 0 & $0(0)$ & $2(100)$ & & & \\
\hline T4a & $02(7.14)$ & 0 & $2(100)$ & $0(0)$ & & & \\
\hline
\end{tabular}

Table 6: Comparison of expression of YAP and nodal metastasis in OSCC

\begin{tabular}{|c|c|c|c|c|c|c|c|}
\hline \multirow[b]{2}{*}{ Nodal status } & \multirow[b]{2}{*}{$N(\%)$} & \multicolumn{3}{|c|}{ YAP expression } & \multirow[b]{2}{*}{ Chi-square value } & \multirow[b]{2}{*}{$p$ value } & \multirow[b]{2}{*}{ Significance } \\
\hline & & Negative & Weak (\%) & Strong (\%) & & & \\
\hline No & $15(53.57)$ & 0 & $12(80)$ & $3(20)$ & 6.0136 & 0.014196 & Significant \\
\hline N1 & $12(42.85)$ & 0 & $4(33.33)$ & $8(66.66)$ & & & \\
\hline N3 & 01 (3.57) & 0 & $1(100)$ & $0(100)$ & & & \\
\hline
\end{tabular}

within the neoplastic population. Ge et al. ${ }^{19}$ found lower indexed YAP expression with greater tumor grade of the head and neck squamous cell carcinoma (HNSCC) with increased expression at the tumor invasive front. Hiemer et al. ${ }^{5}$ observed the emergence of highly enriched nuclear YAP in areas with severe dysplasia, further

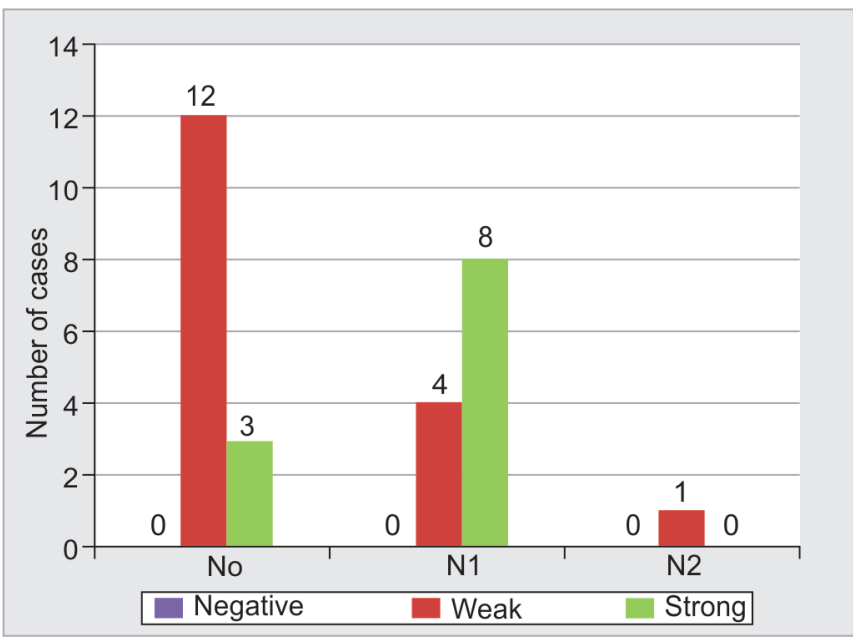

Fig. 5: Comparison of expression of YAP and nodal metastasis in OSCC supporting our findings. The results suggested that predisposition to OSCC may be related to the dysregulation of YAP localization.

The expression of YAP in different stages of OSCC was also compared in this study. Out of the total cases included in the present study, $66.66 \%$ cases of stage I OSCC and $80 \%$ cases of stage II showed a weak expression. Of the total cases of stage III OSCC, $61.53 \%$ cases had a strong expression for YAP. The single case each of stages IVa and IVb had a weak expression. No such parallel study was found in the literature comparing various stages of OSCC with YAP expression. Hence, this parameter of the study could not be compared. However, Ge et al. ${ }^{19}$ observed that nuclear expression of YAP declined with mounting clinical stage in HNSCC.

The expression of YAP in various tumor sizes was compared in OSCC. A strong expression for YAP was observed in one T1 case of OSCC and weak in five cases. Nine cases with T2 demonstrated strong YAP expression, while nine showed weak YAP expression. A strong expression was found in two cases with T3. To the best of our knowledge, no other studies have included a comparison of tumor size and YAP expression in the literature and, thus, we could not compare this parameter as well.

An attempt was made to correspond the YAP expression in different nodal status in metastatic OSCC. A strong association of YAP expression was observed with N1 nodal metastatic OSCC, while a weak expression of YAP was found in N3 metastatic OSCC. 
Ge et al. ${ }^{19}$ have also verified the expression of YAP in the primary HNSCC with nodal metastasis and found an increased cytoplasmic YAP-indexed expression with the occurrence of nodal metastasis in HNSCC.

\section{Conclusions}

In summary, the current study demonstrated that YAP expression significantly increased with successive histologic grades of OSCC, suggesting its possible role as a prognostic marker to detect the severity of OSCC. Taken together our observations indicate that YAP/TAZ contributes in a consequential manner in OSCC oncology. Given that YAP/TAZ are dysregulated during the early onset of OSCC, further discernment of the YAP/TAZ-regulated transcriptional events and amalgamating them to other cancer-related signaling pathways could provide new perspicacity into OSCC. Moreover, novel therapeutic approaches may evolve through the emergence of small molecules duping YAP/TAZ activity, which could help improve the overall threats from this deadly disease. But we counsel future studies on bigger sample size to corroborate our result as our sample size was smaller and we could not include poorly differentiated OSCC in the study group. Future studies are needed to put more cognizances into the role of YAP in prognosticating biological behavior of OSCC.

\section{Clinical Significance}

YAP could be exploited as a prognostic marker for nodal metastasis in OSCC cases.

\section{Ethical Approval}

This article does not contain any studies with human participants or animals performed by any of the authors. All procedures performed in studies were in accordance with the ethical standards of the institutional and/or national research committee.

\section{Informed Consent}

For this type of study, formal consent is not required.

\section{References}

1. Elango JK, Gangadharan P, et al. Trends of head and neck cancers in urban and rural India. Asian Pac J Cancer Prev 2006;7(1):108-112.

2. Sankaranarayanan $\mathrm{R}$, Ramadas $\mathrm{K}$, et al. Effect of screening on oral cancer mortality in Kerala, India: a cluster-randomised controlled trial. Lancet 2005;365(9475):1927-1933. DOI: 10.1016/S01406736(05)66658-5.
3. Manoharan N, Tyagi BB, et al. Cancer incidences in rural Delhi-200405. Asian Pac J Cancer Prev 2010;11(1):73-78.

4. Joshi P, Dutta $S$, et al. Head and neck cancers in developing countries. Rambam Maimonides Med J 2014;5(2):e0009. DOI: 10.5041/ RMMJ.10143.

5. Hiemer SE, Zhang L, et al. A YAP/TAZ-Regulated molecular signature is associated with oral squamous cell carcinoma. Mol Cancer Res 2015;13(6):957-968. DOI: 10.1158/1541-7786.MCR-14-0580.

6. Harvey K, Tapon N. The Salvador-Warts-Hippo pathway: An emerging tumor-suppressor network. Nat Rev Cancer 2007;7(3):182-191. DOI: 10.1038/nrc2070.

7. Harvey KF, Pfleger CM, et al. The Drosophila Mstortholog, hippo, restricts growth and cell proliferation and promotes apoptosis. Cell 2003;114(4):457-467. DOI: 10.1016/S0092-8674(03)00557-9.

8. Huang J, Wu S, et al. The Hippo signaling pathway coordinately regulates cell proliferation and apoptosis by inactivating Yorkie, the Drosophila Homolog of YAP. Cell 2005;122(3):421-434. DOI: 10.1016/ j.cell.2005.06.007.

9. Zhao $B$, Ye X, et al. TEAD mediates YAP-dependent gene induction and growth control. Genes Dev 2008;22(8):1962-1971. DOI: 10.1101/ gad.1664408.

10. Moroishi T, Hansen CG, et al. The emerging roles of YAP and TAZ in cancer. Nat Rev Cancer 2015;15(2):73-79. DOI: 10.1038/nrc3876.

11. Holden JK, Cunningham CN. Targeting the hippo pathway and cancer through the TEAD family of transcription factors. Cancers (Basel) 2018;10(3):81. DOI: 10.3390/cancers10030081.

12. Johnson R, Halder G. The two faces of Hippo: targeting the Hippo pathway for regenerative medicine and cancer treatment. Nat Rev Drug Discov 2014;13(1):63-79. DOI: 10.1038/nrd4161.

13. Watson KL, Justice RW, et al. Drosophila in cancer research: the first fifty tumor suppressor genes. J Cell Sci 1994;18:19-33. DOI: 10.1242/ jcs.1994.Supplement_18.4.

14. Halder G, Johnson RL. Hippo signaling: Growth control and beyond. Development 2011;138(1):9-22. DOI: 10.1242/dev.045500.

15. Lai $\mathrm{D}, \mathrm{Ho} \mathrm{KC}$, et al. Taxol resistance in breast cancer cells is mediated by the hippo pathway component TAZ and its downstream transcriptional targets Cyr61 and CTGF. Cancer Res 2011;71(7):27282738. DOI: 10.1158/0008-5472.CAN-10-2711.

16. Guo L, Teng L. YAP/TAZ for cancer therapy: opportunities and challenges. Int J Oncol 2015;46(4):1444-1452. DOI: 10.3892/ ijo.2015.2877.

17. Zhang $L$, Ye D, et al. Yes-associated protein promotes cell proliferation by activating Fos Related Activator-1 in oral squamous cell carcinoma. Oral Oncol 2011;47(8):693-697. DOI: 10.1016/ j.oraloncology.2011.06.003.

18. Yuan M, Tomlinson V. Yes-associated protein (YAP) functions as tumor suppressor in Breast. Cell Death Differ 2008;15(11):1752-1759. DOI: 10.1038/cdd.2008.108.

19. Ge L, Smail M, et al. Yes-associated protein expression in head and neck squamous cell carcinoma nodal metastasis. Plos One 2011;6(11):e27529. DOI: 10.1371/journal.pone.0027529. 